This item was submitted to Loughborough's Research Repository by the author.

Items in Figshare are protected by copyright, with all rights reserved, unless otherwise indicated.

The collision and snapping of cosmic strings generating spherical impulsive gravitational waves

PLEASE CITE THE PUBLISHED VERSION

LICENCE

CC BY-NC-ND 4.0

REPOSITORY RECORD

Podolsky, J., and J.B. Griffiths. 2019. "The Collision and Snapping of Cosmic Strings Generating Spherical Impulsive Gravitational Waves”. figshare. https://hdl.handle.net/2134/746. 


\title{
The collision and snapping of cosmic strings generating spherical impulsive gravitational waves
}

\author{
J. Podolský* \\ Institute of Theoretical Physics, Charles University, \\ V Holešovičkách 2, 18000 Prague 8, Czech Republic. \\ and J. B. Griffiths ${ }^{\dagger}$ \\ Department of Mathematical Sciences, Loughborough University \\ Loughborough, Leics. LE11 3TU, U.K.
}

January 18, 2000

\begin{abstract}
The Penrose method for constructing spherical impulsive gravitational waves is investigated in detail, including alternative spatial sections and an arbitrary cosmological constant. The resulting waves include those that are generated by a snapping cosmic string. The method is used to construct an explicit exact solution of Einstein's equations describing the collision of two nonaligned cosmic strings in a Minkowski background which snap at their point of collision.
\end{abstract}

\section{Introduction}

Several exact solutions of Einstein's equations have been published which describe an impulsive spherical gravitational wave in a Minkowski background generated, either by a snapping cosmic string (identified by a deficit angle), or by an expanding string inside the sphere.

The first such solution was presented independently by both Gleiser and Pullin [1] and Bičák and Schmidt [2]. The first of these [1] was obtained by pasting two appropriate forms of Minkowski space either side of the spherical wavefront, while the second [2] was obtained as a limiting case of a solution with boost-rotation symmetry. In this case, two null particles recede from a common point generating an impulsive spherical gravitational wave and there are either cosmic strings attaching each particle to infinity, or there is an expanding cosmic string along the axis of symmetry separating the two particles. However, as pointed out by Bičák [3], this solution does not strictly describe a snapping cosmic string, but two semi-infinite cosmic strings initially approaching at the speed of light and then separating again at the instant at which they collide.

In fact a general method for constructing expanding impulsive spherical gravitational waves had previously been presented by Penrose [4]. This involves cutting Minkowski space-time along a null cone and then re-attaching the two pieces with a suitable "warp". The explicit general solution written in a continuous coordinate system was given by Nutku and Penrose [5] and Hogan [6], [7]. The above solutions describing "snapping cosmic strings" are included within this family.

${ }^{*}$ E-mail: Podolsky@mbox.troja.mff.cuni.cz

${ }^{\dagger}$ E-mail: J.B.Griffiths@Lboro.ac.uk 
These solutions have been extended [8] to include a cosmological constant. With this they describe expanding spherical gravitational waves in de Sitter and anti-de Sitter backgrounds. Further, the general class of solutions of this type has been shown [9] to be equivalent to impulsive limits of the Robinson-Trautman type $\mathrm{N}$ class of solutions. Finally, it may be mentioned that Hortaçsu and his colleagues [10]-[12] have considered aspects of particle creation in these backgrounds.

It may be noted that Hogan [13] has also considered imploding-exploding gravitational waves. In this he has simply attached an impulsive wave on a past null cone to one on a future null cone without considering the necessary sources of the wave. Outside the cone, a snapping string must evolve continuously. However, this construction does permit a string inside an expanding cone to be different from one inside the prior imploding cone, although the singular event at which the two cones join would then require some physical justification.

The purpose of the present paper is to describe the Penrose method and its interpretation in detail and in full generality, using alternative spatial sections and including an arbitrary cosmological constant. This will enable us to construct an exact solution which describes the gravitational wave that would be generated by the collision (and consequent breaking) of a pair of moving cosmic strings in a Minkowski background. Such a situation was outlined in [5]. The explicit solution is given in section VI.

\section{The explicit Penrose construction}

As mentioned above, Penrose [4] has described a "cut and paste" method for constructing an expanding spherical gravitational wave in a Minkowski background. In this method, appropriate junction conditions across the null cone guarantee that the vacuum field equations are satisfied. However, there is an impulsive component in the Weyl tensor representing an impulsive gravitational wave located on this null cone.

The above procedure will now be performed explicitly. However, for later convenience, we will derive a more general form of the solution for an impulsive spherical wave in a Minkowski background than that outlined in [4]. In addition, we will also include a cosmological constant $\Lambda$, so that the resulting solutions will also describe expanding spherical impulsive waves in de Sitter and anti-de Sitter backgrounds. In this approach, it is convenient to start with the line element for space-times of constant curvature in the manifestly conformally flat form

$$
\mathrm{d} s^{2}=\frac{2 \mathrm{~d} \eta \mathrm{d} \bar{\eta}-2 \mathrm{~d} u \mathrm{~d} v}{\left[1+\frac{1}{6} \Lambda(\eta \bar{\eta}-u v)\right]^{2}}
$$

where the relation to conformal cartesian coordinates is $\eta=\frac{1}{\sqrt{2}}(x+i y), u=\frac{1}{\sqrt{2}}(t+z)$ and $v=\frac{1}{\sqrt{2}}(t-z)$.

We may now perform the transformation

$$
\begin{aligned}
v & =\frac{V}{p}-\epsilon U, \\
u & =Z \bar{Z} \frac{V}{p}-U, \\
\eta & =\frac{V}{p} Z,
\end{aligned}
$$

where

$$
p=1+\epsilon Z \bar{Z}, \quad \epsilon=-1,0,1 .
$$

The parameter $\epsilon$ is related to the Gaussian curvature of the 2-surfaces given by $U=$ const., $V=$ const. [9]. With this, the metric (1) becomes

$$
\mathrm{d} s^{2}=\frac{2 \frac{V^{2}}{p^{2}} \mathrm{~d} Z \mathrm{~d} \bar{Z}+2 \mathrm{~d} U \mathrm{~d} V-2 \epsilon \mathrm{d} U^{2}}{\left[1+\frac{1}{6} \Lambda U(V-\epsilon U)\right]^{2}} .
$$


Let us also consider the alternative, and more involved, transformation given by

$$
\begin{aligned}
& v=A V-D U, \\
& u=B V-E U, \\
& \eta=C V-F U,
\end{aligned}
$$

where

$$
\begin{aligned}
& A=\frac{1}{p\left|h^{\prime}\right|}, \quad B=\frac{|h|^{2}}{p\left|h^{\prime}\right|}, \quad C=\frac{h}{p\left|h^{\prime}\right|}, \\
& D=\frac{1}{\left|h^{\prime}\right|}\left\{\frac{p}{4}\left|\frac{h^{\prime \prime}}{h^{\prime}}\right|^{2}+\epsilon\left[1+\frac{Z}{2} \frac{h^{\prime \prime}}{h^{\prime}}+\frac{\bar{Z}}{2} \frac{\bar{h}^{\prime \prime}}{\bar{h}^{\prime}}\right]\right\}, \\
& E=\frac{|h|^{2}}{\left|h^{\prime}\right|}\left\{\frac{p}{4}\left|\frac{h^{\prime \prime}}{h^{\prime}}-2 \frac{h^{\prime}}{h}\right|^{2}+\epsilon\left[1+\frac{Z}{2}\left(\frac{h^{\prime \prime}}{h^{\prime}}-2 \frac{h^{\prime}}{h}\right)+\frac{\bar{Z}}{2}\left(\frac{\bar{h}^{\prime \prime}}{\bar{h}^{\prime}}-2 \frac{\bar{h}^{\prime}}{\bar{h}}\right)\right]\right\}, \\
& F=\frac{h}{\left|h^{\prime}\right|}\left\{\frac{p}{4}\left(\frac{h^{\prime \prime}}{h^{\prime}}-2 \frac{h^{\prime}}{h}\right) \frac{\bar{h}^{\prime \prime}}{\bar{h}^{\prime}}+\epsilon\left[1+\frac{Z}{2}\left(\frac{h^{\prime \prime}}{h^{\prime}}-2 \frac{h^{\prime}}{h}\right)+\frac{\bar{Z}}{2} \frac{\bar{h}^{\prime \prime}}{\bar{h}^{\prime}}\right]\right\},
\end{aligned}
$$

$h=h(Z)$ is an arbitrary holomorphic function (apart from its singular regions), and the derivative with respect to $Z$ is denoted by a prime. With this, the Minkowski, de Sitter and antide Sitter metric (1) becomes

$$
\mathrm{d} s^{2}=\frac{2\left|\frac{V}{p} \mathrm{~d} Z+U p \bar{H} \mathrm{~d} \bar{Z}\right|^{2}+2 \mathrm{~d} U \mathrm{~d} V-2 \epsilon \mathrm{d} U^{2}}{\left[1+\frac{1}{6} \Lambda U(V-\epsilon U)\right]^{2}},
$$

where $2 H(Z)=\beta^{\prime}-\frac{1}{2} \beta^{2}, \beta=\alpha^{\prime} / \alpha$ and $\alpha=h^{\prime}$. Thus,

$$
H(Z)=\frac{1}{2}\left[\frac{h^{\prime \prime \prime}}{h^{\prime}}-\frac{3}{2}\left(\frac{h^{\prime \prime}}{h^{\prime}}\right)^{2}\right]
$$

is the Schwarzian derivative. Notice that the transformation (5) and (6) reduces to (2) when $h=Z$.

In the coordinates of both line elements (4) and (7), the null hypersurface $U=0$ represents a null cone (an expanding sphere) $\eta \bar{\eta}-u v=0$ in the background. Moreover, the reduced 2-metrics on this cone are identical. Following Penrose's "cut and paste" method [4], we may take the line element (4) for $U<0$ and attach this to (7) for $U>0$. The resulting line element can then be written in the combined form

$$
\mathrm{d} s^{2}=\frac{2\left|\frac{V}{p} \mathrm{~d} Z+U \Theta(U) p \bar{H} \mathrm{~d} \bar{Z}\right|^{2}+2 \mathrm{~d} U \mathrm{~d} V-2 \epsilon \mathrm{d} U^{2}}{\left[1+\frac{1}{6} \Lambda U(V-\epsilon U)\right]^{2}},
$$

where $\Theta(U)$ is the Heaviside step function. This combined metric, which was presented for a Minkowski background in [5]-[7], with a cosmological constant in [8], and in the most general form in [9], is explicitly continuous everywhere, including across the null hypersurface $U=0$. However, the discontinuity in the derivatives of the metric yields impulsive components in the curvature tensor proportional to the Dirac $\delta$-function.

It may be observed that the Penrose junction conditions can be obtained by comparing the transformations $(2)$ at $U=0_{-}$with (5-6) at $U=0_{+}$. This gives the identification

$$
(Z, \bar{Z}, V, U=0)_{M^{-}}=\left(h(Z), \bar{h}(\bar{Z}), \frac{1+\epsilon h \bar{h}}{1+\epsilon Z \bar{Z}} \frac{V}{\left|h^{\prime}\right|}, U=0\right)_{M^{+}},
$$


where the space-time has been divided into two halves $M^{-}(U<0)$ inside the null cone, and $M^{+}(U>0)$ outside.

Labelling coordinates $\left(x^{1}, x^{2}, x^{3}, x^{4}\right)=(Z, \bar{Z}, V, U)$, and using the tetrad

$$
\begin{aligned}
k^{\mu} & =\left[1+\frac{1}{6} \Lambda U(V-\epsilon U)\right]^{2} \delta_{3}^{\mu}, \\
\ell^{\mu} & =-\delta_{4}^{\mu}-\epsilon \delta_{3}^{\mu}, \\
m^{\mu} & =p^{2} \frac{1+\frac{1}{6} \Lambda U(V-\epsilon U)}{V^{2}-p^{4} U^{2} \Theta(U) H \bar{H}}\left(\frac{V}{p} \delta_{2}^{\mu}-p U \Theta(U) \bar{H} \delta_{1}^{\mu}\right),
\end{aligned}
$$

the non-zero components of the curvature tensor for the line element (9) can be shown to be given by

$$
\Psi_{4}=\frac{p^{2} H}{V} \delta(U), \quad \Phi_{22}=\frac{p^{4} H \bar{H}}{V^{2}} U \delta(U) .
$$

This indicates an impulsive gravitational wave component. It also confirms that the space-time is vacuum everywhere except on the wave surface at $V=0$ and at possible singularities of the function $p^{2} H(Z)$.

It may be observed that the metric (4) contains a coordinate singularity at $V=0$. However, this becomes a physical singularity on the wave surface $U=0$ in the above construction. Unfortunately, for $\epsilon=0, V=0$ is a singular null line on the surface $U=0$. In fact, as seen from (2), this is a common line $(x=y=0, z=t)$ to all the null cones $U=$ const. Thus, for $\epsilon=0$, there is a singular line on the wave surface where $V=0$, in addition to possible singular points of $H$. For a physical interpretation of these solutions, it would be better to remove the singularity $V=0$ from the wavefront. This can be achieved by considering solutions with $\epsilon \neq 0$. For these cases, as observed by Hogan [7], the family of null cones $U=$ const. have vertices on a timelike line $(x=y=z=0)$ if $\epsilon=1$ and on a spacelike line $(x=y=t=0)$ if $\epsilon=-1$. For either of these cases, the singularity at $V=0$ appears only at the vertex of the null cone $x=y=z=t=0$, which may be considered as the "origin" of the spherical wave.

\section{A geometrical description of the impulse}

Significantly, the construction of the spherical impulsive wave as described above admits an interesting geometrical interpretation. Specifically, the ratio

$$
\xi \equiv \frac{\eta}{v}=\frac{x+i y}{t-z}
$$

is the well known relation for a stereographic (one-to-one) correspondence between a sphere and an Argand plane by a projection from the North pole onto a plane through the equator (see chapter 1 of [14]). This permits us to represent the wave surface $U=0$ (taken at a typical or rescaled time $t=1$ ) either as a Riemann sphere, or as its associated complex plane. Conversely, any point $\xi$ on the complex plane, taken as $z=0$, identifies a unique point $P$ on the sphere with coordinates

$$
x=\frac{\xi+\bar{\xi}}{1+\xi \bar{\xi}}, \quad y=\frac{i(\bar{\xi}-\xi)}{1+\xi \bar{\xi}}, \quad z=\frac{\xi \bar{\xi}-1}{\xi \bar{\xi}+1} .
$$

In terms of standard spherical coordinates, $\xi=\cot \frac{\theta}{2} e^{i \phi}$.

We may also recall some important properties of the stereographic projection. Any circle on the Riemann sphere maps to a circle in the complex Argand plane, and vice versa. As a special case, any circle passing through the North pole of the Riemann sphere maps to a straight line in the complex plane. A great circle which passes through the North pole maps to a straight line through the origin of the plane. We also note that the stereographic projection is conformal, i.e. angle preserving. 
Returning to the impulsive spherical wave, we observe from (2) and (5-6) that on $U=0$

$$
\xi=\left\{\begin{array}{lll}
Z & \text { for } & U=0_{-} \\
h(Z) & \text { for } & U=0_{+}
\end{array}\right.
$$

This permits us to represent the Penrose junction condition (10) across the wave surface as a mapping on the complex Argand plane $Z \rightarrow h(Z)$. This is equivalent to mapping points $P_{-}$on the "inside" of the wave surface to the identified points $P_{+}$on the "outside", see Fig. 1.

Normally, we will assume that the "inside" represented by $Z$ covers the complete sphere, but the function $h(Z)$ will not generally cover the entire sphere on the "outside". The restrictions on the range of the function $h(Z)$ together with its specific character will correspond to particular physical situations as will be described below.

For the sake of completeness, the results of these two sections have been given with an arbitrary cosmological constant included. However, for the remainder of this paper, we will only consider the case when $\Lambda=0$.

\section{A snapping cosmic string}

The utility of the above geometrical approach may be demonstrated by the simplest and physically interesting case of an impulsive spherical wave generated by a "snapping cosmic string". Without loss of generality, the string may be taken to be located along the $z$-axis perpendicular to the above complex plane.

It may be noted that the initial Gleiser-Pullin [1] solution describing this situation was presented in a slightly different form to that given above. Essentially, it employed two different constant non-zero values of $H$ in the regions inside and outside the spherical wave. This is effectively equivalent to taking $\xi=e^{Z}$ for $U<0$ and $\xi=e^{(1-\delta) Z}$ for $U>0$, where $\delta$ is a real positive constant.

Here we will describe the same solution. However we will use the above notation as also presented by Nutku and Penrose [5]. Taking $\Lambda=0$, the line element (9) becomes

$$
\mathrm{d} s^{2}=2\left|\frac{V}{p} \mathrm{~d} Z+U \Theta(U) p \bar{H} \mathrm{~d} \bar{Z}\right|^{2}+2 \mathrm{~d} U \mathrm{~d} V-2 \epsilon \mathrm{d} U^{2} .
$$

where $p$ and $\epsilon$ are given by (3).

According to the above construction (13), in the interior region $U<0$, the complete spherical surface is covered by $\xi=Z$. It is then appropriate to represent the outer region $U>0$ by $\xi=h_{1}(Z)$ where

$$
h_{1}(Z)=Z^{1-\delta}
$$

Putting $Z=|Z| e^{i \phi}$ where $\phi \in[-\pi, \pi), h_{1}(Z)$ covers the plane minus a wedge, e.g. $\arg h_{1}(Z) \in$ $[-(1-\delta) \pi,(1-\delta) \pi]$. This represents Minkowski space with a deficit angle $2 \pi \delta$ and may be considered to describe a cosmic string in the region outside the spherical wave as shown in Fig. 2. (Alternatively, if $\delta<0$ and the exterior region may be taken to be complete and the range of $\phi$ reduced so that the deficit angle representing the string appears inside the sphere.) Outside the spherical wavefront, the string may be taken to be located along the axis $\eta=0$. Then, by putting $\eta=|\eta| e^{i \phi}$, it may be observed from (5) that, for the particular function $h_{1}(Z)$, $\arg \eta=\arg h_{1}$. Thus, the deficit angle is constant along the length of the string, corresponding to a constant tension.

For the case when $h=h_{1}(Z)$, the metric function $H_{1}(Z)$ in (14) is given by

$$
H_{1}=\frac{\frac{1}{2} \delta\left(1-\frac{1}{2} \delta\right)}{Z^{2}} .
$$

This indicates a singular point at $Z=0$, which is located at the South pole of the spherical wavefront. However, there is an additional singularity at $V=0$ which, for $\epsilon=0$, is located at 
the North pole at any time $t>0$. As suggested at the end of section II, a much better physical interpretation can be given to the $\epsilon \neq 0$ solutions of the form (14). For $\epsilon= \pm 1$, the physical singularity at $V=0$ appears only in the vertex of the conical impulsive surface $U=0$ at $t=0$. Moreover, according to (11) and (16)

$$
\Phi_{22} \sim \frac{(1+\epsilon Z \bar{Z})^{4}}{(Z \bar{Z})^{2}} \frac{U \delta(U)}{V^{2}} .
$$

Therefore, there is a divergence not only at the South pole where $Z=0$ given by $\Phi_{22} \sim|Z|^{-4}$, but also at the North pole where $Z=\infty$ given by $\Phi_{22} \sim|Z|^{4}$. Both these divergences are equivalent and indicate particles of equal "mass" at the two ends of the string.

\section{$5 \quad$ Lorentz transformations}

At this point, let us consider a space-time given by the line element (14) for some particular function $H$ and observe that it can best be interpreted by first calculating the associated function $h(Z)$. This can be achieved by integrating the three first order equations following (7). However, the resulting function is not unique as it contains three complex constants of integration. In fact, this arbitrariness corresponds to the freedom associated with the Lorentz transformations

$$
Z \rightarrow h(Z)=\frac{a Z+b}{c Z+d}
$$

where $a, b, c, d$ are arbitrary complex constants satisfying the complex condition $a d-b c=1$. It is well known that this is the most general global holomorphic (i.e. analytic and conformal) transformation of the Riemann sphere to itself (see chapter 1 of [14]). It may also be noted that the Schwarzian derivative (8), i.e. $H(Z)$, is invariant under this transformation.

In particular, a rotation about an arbitrary direction can be given by

$$
h_{r}(Z)=\frac{Z e^{i \psi} \cos \frac{\theta}{2}-\sin \frac{\theta}{2}}{Z e^{i \psi} \sin \frac{\theta}{2}+\cos \frac{\theta}{2}} e^{i \phi},
$$

where $\theta, \phi, \psi$ are the Euler angles. We will adopt the following construction: first we perform a rotation through $\psi$ about the $z$-axis, then we rotate through $\theta$ about the original $y$-direction, and finally we perform a rotation through $\phi$ about the original $z$-axis. This moves the North pole to a point on the sphere given by the spherical coordinates $\theta, \phi$.

Of the remaining Lorentz transformations, a boost in the $z$-direction with velocity $v$ is given simply by

$$
h_{b}(Z)=w Z,
$$

where $w=\sqrt{(1+v) /(1-v)}$, which corresponds to a uniform expansion in the complex plane. It leaves invariant both the poles of the Riemann sphere. Also, the null rotation $h_{n}(Z)=Z-Z_{0}$, where $Z_{0}$ is complex, is a uniform translation in the complex plane. It leaves invariant only the North pole of the Riemann sphere. These are well described with useful pictures in chapter 1 of [14].

\section{The collision of cosmic strings}

By combining operations of the type given in (15), which introduces a deficit angle, with suitable Lorentz transformations, we can generate much more general solutions with predetermined physical properties. In particular, we can use this technique to construct an explicit solution describing the situation outlined by Nutku and Penrose [5] in which two cosmic strings collide and split generating an impulsive spherical gravitational wave. 
It is assumed that two strings are initially moving with constant velocity and approach each other. Of course, provided they are not parallel, it is always possible to make a Lorentz transformation to a frame of reference in which the two cosmic strings are orthogonal. There is therefore no loss of generality in considering only orthogonal strings. It is also assumed that both strings snap at their point of intersection and that the snapped ends propagate along the length of the string at the speed of light. This will generate an impulsive spherical gravitational wave.

We start with the simplest situation in which two orthogonal strings approach each other with a negligible velocity. The solution for such a situation can be constructed as follows.

Starting with (15), we first apply a rotation (17) with $\psi=\theta=\phi=\pi / 2$. This leads to $\left(i h_{1}-1\right) /\left(h_{1}-i\right)$, which represents a cosmic string located along the $y$-axis. Then we introduce another string in the same way as in (15) by taking the $(1-\varepsilon)$-th power. This removes a wedge which represents a second string that is located along the $z$-axis and has deficit angle $2 \pi \varepsilon$. The final mapping is given by

$$
h_{2}(Z)=\left(\frac{i Z^{1-\delta}-1}{Z^{1-\delta}-i}\right)^{1-\varepsilon}
$$

This solution describes two snapped orthogonal cosmic strings which were at "relative rest" initially. The corresponding function $H_{2}(Z)$ has the form

$$
H_{2}(Z)=\frac{\frac{1}{2} \delta\left(1-\frac{1}{2} \delta\right)}{Z^{2}}-\frac{\frac{1}{2} \varepsilon\left(1-\frac{1}{2} \varepsilon\right) 4(1-\delta)^{2} Z^{-2 \delta}}{\left(Z^{1-\delta}+i\right)^{2}\left(Z^{1-\delta}-i\right)^{2}} .
$$

The solution is visualized in Fig. 3. On the left there is the complex function $h_{2}(Z)$ in the complex Argand plane, on the right the corresponding Riemann sphere obtained using the stereographic identification. As can be seen, there are two perpendicular wedge cuts on the sphere indicating that the flat space outside the spherical impulsive gravitational wave contains two orthogonal pairs of cosmic strings. This solution thus represents two cosmic strings which snapped at the initial time $t=0$ and at their point of intersection.

We can now similarly construct a more general solution describing a situation in which the two cosmic strings were in finite relative motion before the collision. In this case, the four snapped half-strings are also in relative translational motion. The more involved construction of such a solution is shown in Figs. 4 and 5.

We start with the initial cut $h_{1}(Z)=Z^{1-\delta}$ given by (15) introducing the first string with deficit angle $2 \pi \delta$ along the $z$-axis as indicated in Fig. 2. A subsequent rotation (17) with $\theta=\phi=\pi / 2, \psi=\pi$ places the initial poles $Z=0$ and $Z=\infty$ on the $y$-axis with the cut passing through the South pole. The corresponding function $h_{a}(Z)=i\left(h_{1}+1\right) /\left(h_{1}-1\right)$ is illustrated in the complex Argand plane in Fig. 4 (a) and on the Riemann sphere in Fig. 5 (a). Next we make a boost (18) with $w=w_{1}$ in the $z$-direction as shown in Fig. 4 (b) and Fig. 5 (b). Then another rotation (17) with $\theta=-\pi / 2, \phi=\psi=0$ about the $y$-axis leads to

$$
h_{c}(Z)=-\frac{\left(w_{1}-i\right) Z^{1-\delta}+\left(w_{1}+i\right)}{\left(w_{1}+i\right) Z^{1-\delta}+\left(w_{1}-i\right)},
$$

and puts the poles and the cut along the $z=0$ plane, symmetric about the $x$-axis, see Fig. 4 (c) and Fig. 5 (c). At this point we make a second cut from the North to South poles through the negative $x$-axis by taking $h_{c}^{1-\varepsilon}$. This introduces the second string with deficit angle $2 \pi \varepsilon$ along the $z$-axis as indicated in Fig. 4 (d) and Fig. 5 (d). (Note that the function $h_{c}^{1-\varepsilon}$ for $w_{1}=1$ exactly reduces to the previous form (19) with the poles located at $h_{c}(0)=\left(i+w_{1}\right) /\left(i-w_{1}\right)=-i$ and $h_{c}(\infty)=\bar{h}_{c}(0)=i$.) The next step is to make a rotation about the $y$-axis using $\theta=\pi / 2$, $\phi=\psi=0$ (see Fig. 4 (e) and Fig. 5 (e)). Finally we perform a second boost $w_{2}$ in the negative $z$-direction. We thus obtain

$$
h_{2}(Z)=w_{2} \frac{h_{c}^{1-\varepsilon}-1}{h_{c}^{1-\varepsilon}+1} .
$$


This expression represents one cut through the North pole parallel to the $x$-direction, and a second cut through the South pole parallel to the $y$-direction, but with both pairs of strings separated either side of the plane $z=0$ as shown in Fig. 4 (f) and Fig. 5 (f). The resulting metric function using (22) with (21) is given by

$$
H_{2}(Z)=\frac{\frac{1}{2} \delta\left(1-\frac{1}{2} \delta\right)}{Z^{2}}-\frac{\frac{1}{2} \varepsilon\left(1-\frac{1}{2} \varepsilon\right) 16 w_{1}^{2}(1-\delta)^{2} Z^{-2 \delta}}{\left[\left(w_{1}+i\right) Z^{1-\delta}+\left(w_{1}-i\right)\right]^{2}\left[\left(w_{1}-i\right) Z^{1-\delta}+\left(w_{1}+i\right)\right]^{2}} .
$$

This is the explicit solution which describes the collision and consequent snapping of two orthogonal cosmic strings as outlined by Nutku and Penrose [5].

It may be noted that there exist null "particles" at the ends of the four semi-infinite strings. These are located on the spherical impulsive wave surface at points given by $\xi_{1+}=h_{2}\left(h_{c}(Z=\right.$ $0))=i w_{2} \tan \left[(1-\varepsilon)\left(\phi_{1}-\pi / 2\right)\right]$, where $\phi_{1}=\arg \left(w_{1}+i\right)$, i.e. $\cot \phi_{1}=w_{1}, \xi_{2+}=h_{2}\left(h_{c}(Z=\infty)\right)=$ $\bar{\xi}_{1+}$, and $\xi_{3+}=h_{2}\left(h_{c}=0\right)=-w_{2}, \xi_{4+}=h_{2}\left(h_{c}=\infty\right)=w_{2}$. These two pairs of points describe the four singular points in the complex Argand plane of Fig. 4 (f). Using the stereographic relation to standard spherical coordinates, $\xi=\cot (\theta / 2) e^{i \phi}$, the corresponding four points on the Riemann sphere - the ends of the two cuts of Fig. 5 (f) - have $\cot \left(\theta_{1} / 2\right)=\left|\xi_{1+}\right|=\left|\xi_{2+}\right|$ and $\cot \left(\theta_{2} / 2\right)=\left|\xi_{3+}\right|=\left|\xi_{4+}\right|$. The values of $\theta_{1}$ and $\theta_{2}$ can be made arbitrary by a suitable choice of the boost parameters $w_{1}$ and $w_{2}$. Consequently, the cuts representing the strings can be distributed arbitrarily over the spherical impulsive wave (parallel to the $x$ and $y$-directions in our construction). It is natural to consider a geometrically privileged situation in which the two cuts are distributed symmetrically, as indicted in Fig. 5 (f). Obviously, this is given by the condition $\theta_{1}+\theta_{2}=\pi$ implying $\cot \left(\theta_{1} / 2\right)=\tan \left(\theta_{2} / 2\right)$, i.e. $\left|\xi_{1+}\right|\left|\xi_{3+}\right|=1$. Therefore, the symmetry condition can be expressed as

$$
w_{2}=\sqrt{\cot \left[(1-\varepsilon)\left(\pi / 2-\phi_{1}\right)\right]}, \text { where } \cot \phi_{1}=w_{1} .
$$

For a negligible $\varepsilon, w_{2} \approx 1 / \sqrt{w_{1}}$.

Note that the above choice of the boost parameters in our construction results in a geometrically symmetric situation in which the two semi-infinite parts of the first snapped cosmic string are parallel to the $y$-axis and propagate in the positive $z$-direction with speed $\cos \theta_{1} \equiv\left|v_{2}\right|$. Also, the two semi-infinite parts of the second string are parallel to the $x$-axis and move in the negative $z$-direction with the same speed $-\left|v_{2}\right|$. For $\varepsilon=\delta$ the strings have equal tension, and the geometrically symmetric situation corresponds to a choice of physically privileged coordinates in which the geometrical origin of the impulse coincides with the centre of mass of the colliding system.

Finally, it can be observed that the solution describing the collision and snapping of two cosmic strings can alternatively and equivalently be constructed if, starting again with (15), we perform the rotation (17) using $\theta=\phi=\pi / 2, \psi=0$ in step (a), and the rotation $\theta=\pi / 2$, $\phi=\psi=0$ in step (c). Taking the other four steps to be the same as in the previous construction shown in Fig. 4 and Fig. 5, this leads to

$$
h_{c}(Z)=\frac{\left(w_{1}+i\right) Z^{1-\delta}-\left(w_{1}-i\right)}{\left(w_{1}-i\right) Z^{1-\delta}-\left(w_{1}+i\right)} .
$$

This results in the solution given by (22) with (25) presented in Fig. 6. Again, there is a cut through the North pole, parallel to the $x$-direction and a second cut through the South pole, parallel to the $y$-direction, but these cuts are smaller than in Fig. 5 (f). The resulting metric function is given by

$$
H_{2}(Z)=\frac{\frac{1}{2} \delta\left(1-\frac{1}{2} \delta\right)}{Z^{2}}-\frac{\frac{1}{2} \varepsilon\left(1-\frac{1}{2} \varepsilon\right) 16 w_{1}^{2}(1-\delta)^{2} Z^{-2 \delta}}{\left[\left(w_{1}+i\right) Z^{1-\delta}-\left(w_{1}-i\right)\right]^{2}\left[\left(w_{1}-i\right) Z^{1-\delta}-\left(w_{1}+i\right)\right]^{2}} .
$$

The two cuts representing the strings are distributed symmetrically along the spherical impulsive wave if $w_{2}=\sqrt{\cot \left[(1-\varepsilon) \phi_{1}\right]}$. 


\section{Conclusions}

We have described the Penrose "cut and paste" method in detail and full generality, emphasizing its geometrical properties and physical interpretation. Using this, we have explicitly constructed the exact solution which describes two colliding cosmic strings which snap at the point of their intersection. The ends of each semi-infinite string are singular points (interpreted as null particles) located on a sphere which is expanding with the speed of light. This generates a spherical impulsive gravitational wave.

\section{Acknowledgments}

This work was supported by a visiting fellowship from the Royal Society and, in part, by the grant GACR-202/99/0261 of the Czech Republic.

\section{References}

[1] Gleiser R and Pullin J 1989 Class. Quantum Grav. 6 L141

[2] Bičák J and Schmidt B 1989 Class. Quantum Grav. 61547

[3] Bičák J 1990 Astron. Nachr. 311189

[4] Penrose R 1972 General Relativity ed L O'Raifeartaigh (Oxford: Clarendon)

[5] Nutku Y and Penrose R 1992 Twistor Newsletter No. 34, 11 May, 9

[6] Hogan P A 1993 Phys. Rev. Lett. 70117

[7] Hogan P A 1994 Phys. Rev. D 496521

[8] Hogan P A 1992 Phys. Lett. A 17121

[9] Podolský J and Griffiths J B 1999 Class. Quantum Grav. 162937

[10] Hortaçsu M 1990 Class. Quantum Grav. 7 L165

[11] Hortaçsu M 1996 Class. Quantum Grav. 132683

[12] Bilge A H, Hortaçsu H and Özdemir N 1996 Gen. Relativ. Gravit. 28511

[13] Hogan P A 1995 Lett. Math. Phys. 35277

[14] Penrose R and Rindler W 1984 Spinors and Space-time, vol.1 (Cambridge: Cambridge University Press) 


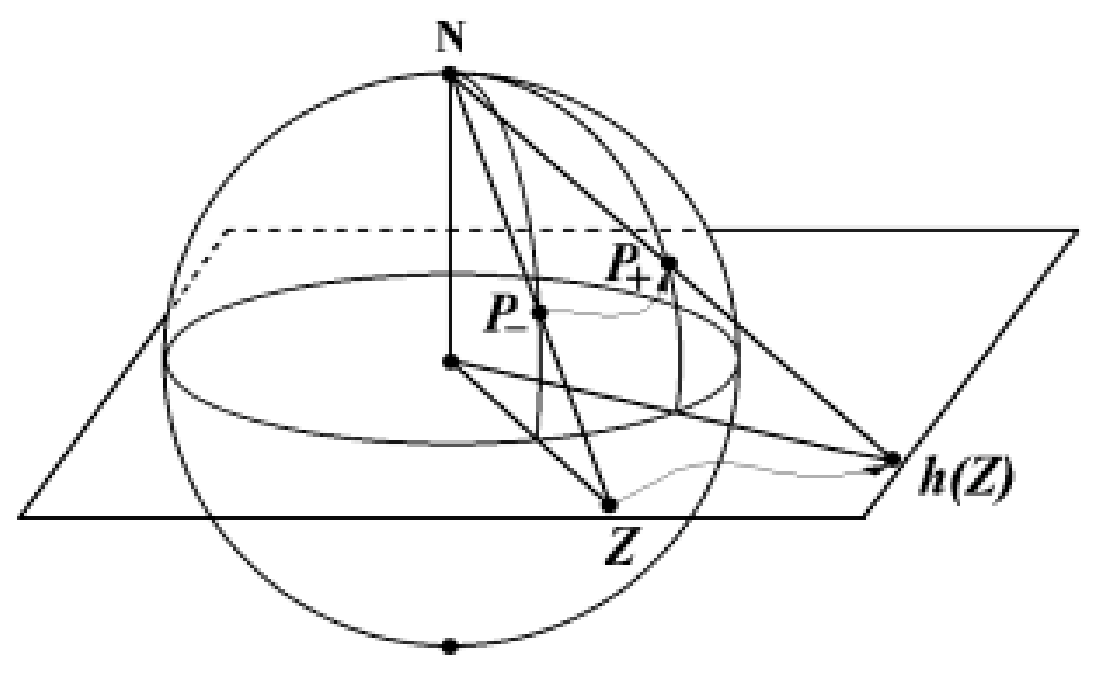

Figure 1: The stereographic correspondence between the Riemann sphere and the complex Argand plane enables a geometrical description of the Penrose junction conditions. Mapping in the complex plane $Z \rightarrow h(Z)$ is equivalent to mapping points $P_{-}$inside the impulsive spherical surface to the corresponding points $P_{+}$outside.

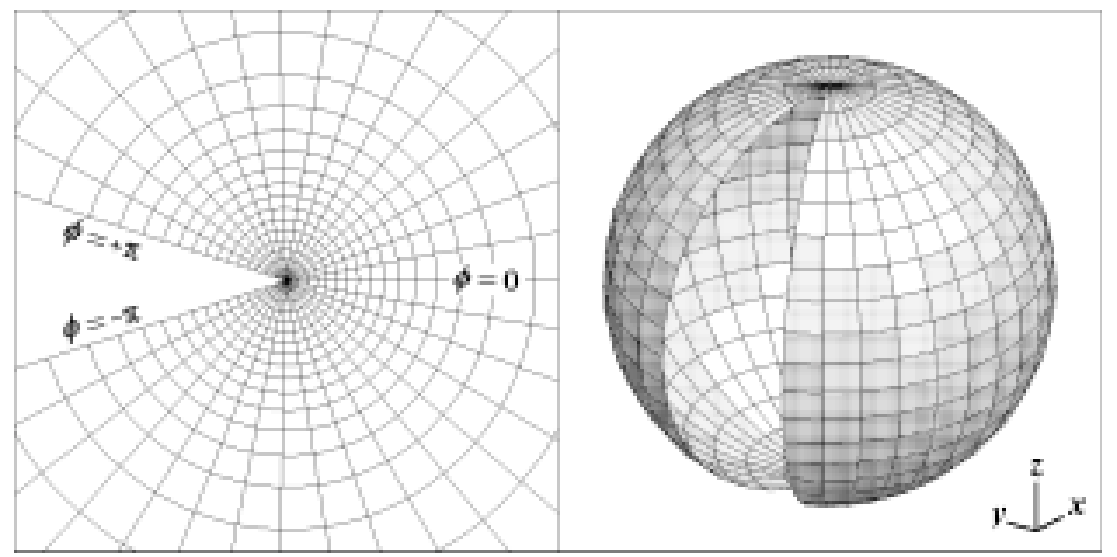

Figure 2: The complex mapping $Z \rightarrow h_{1}(Z)=Z^{1-\delta}$ (left) corresponds to the cut through the Riemann sphere (right). This represents Minkowski space with a deficit angle $2 \pi \delta$ outside the expanding spherical impulse, i.e. a "snapping cosmic string" along the $z$-axis. 


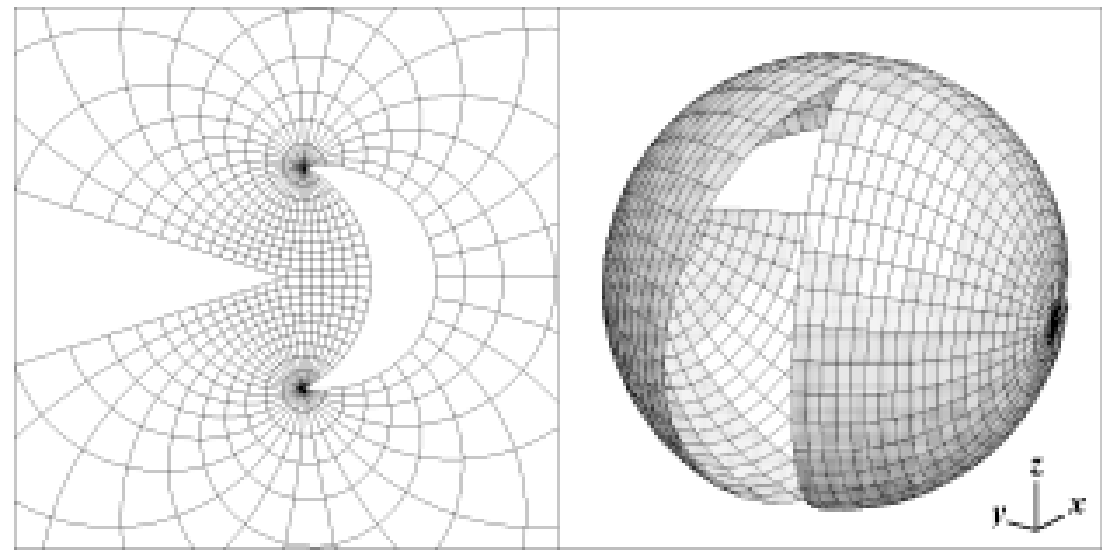

Figure 3: The mapping $Z \rightarrow h_{2}(Z)$ given by Eq. (19) in the complex Argand plane (left) corresponds to two orthogonal cuts through the Riemann sphere (right). This describes two cosmic strings along the $y$ and $z$-axes which are "snapped" at their point of intersection.

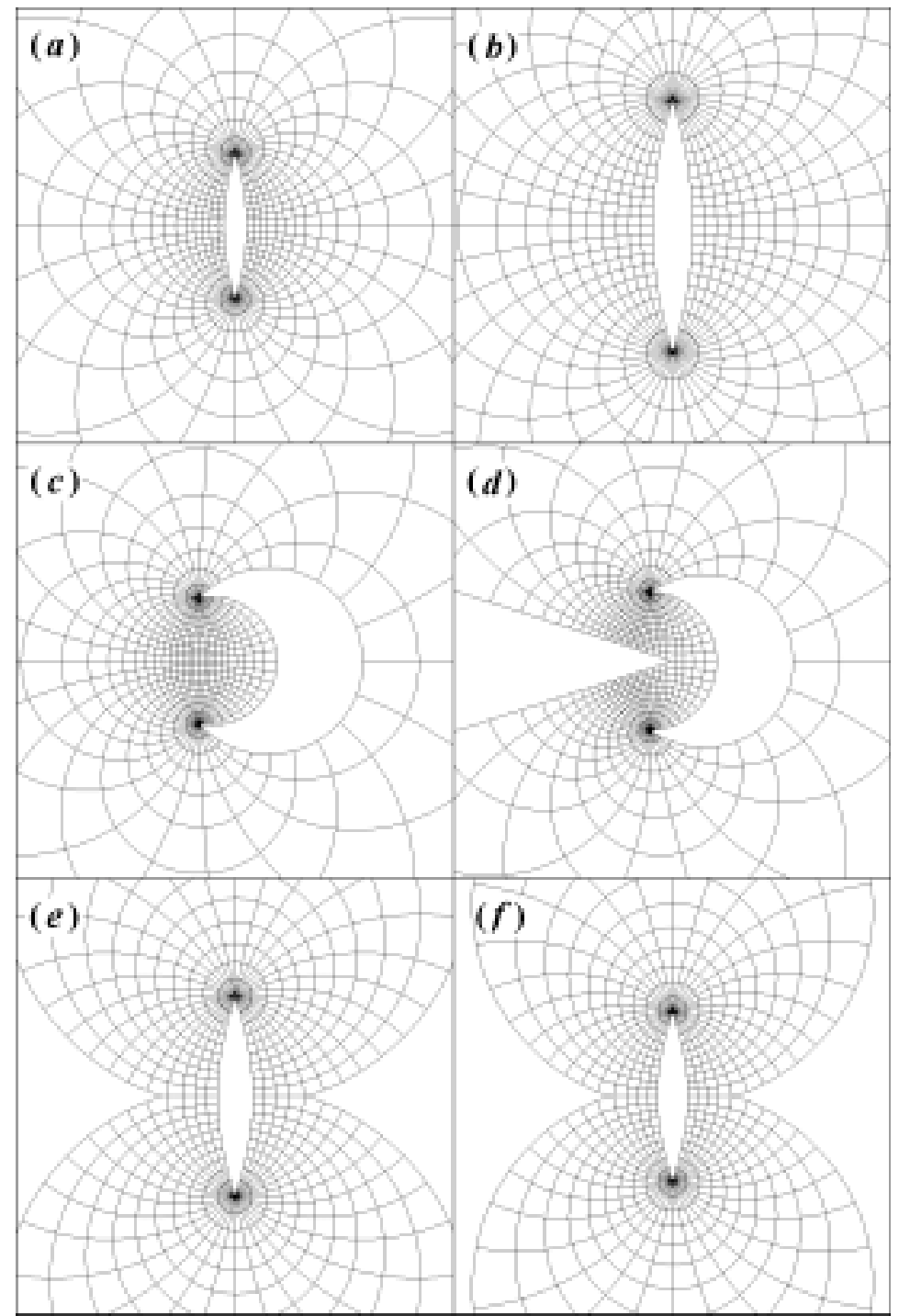

Figure 4: Representation in the complex Argand plane of the construction of the solution for two cosmic strings parallel to the $x$ and $y$-axes and moving apart in the positive and negative $z$-directions. 


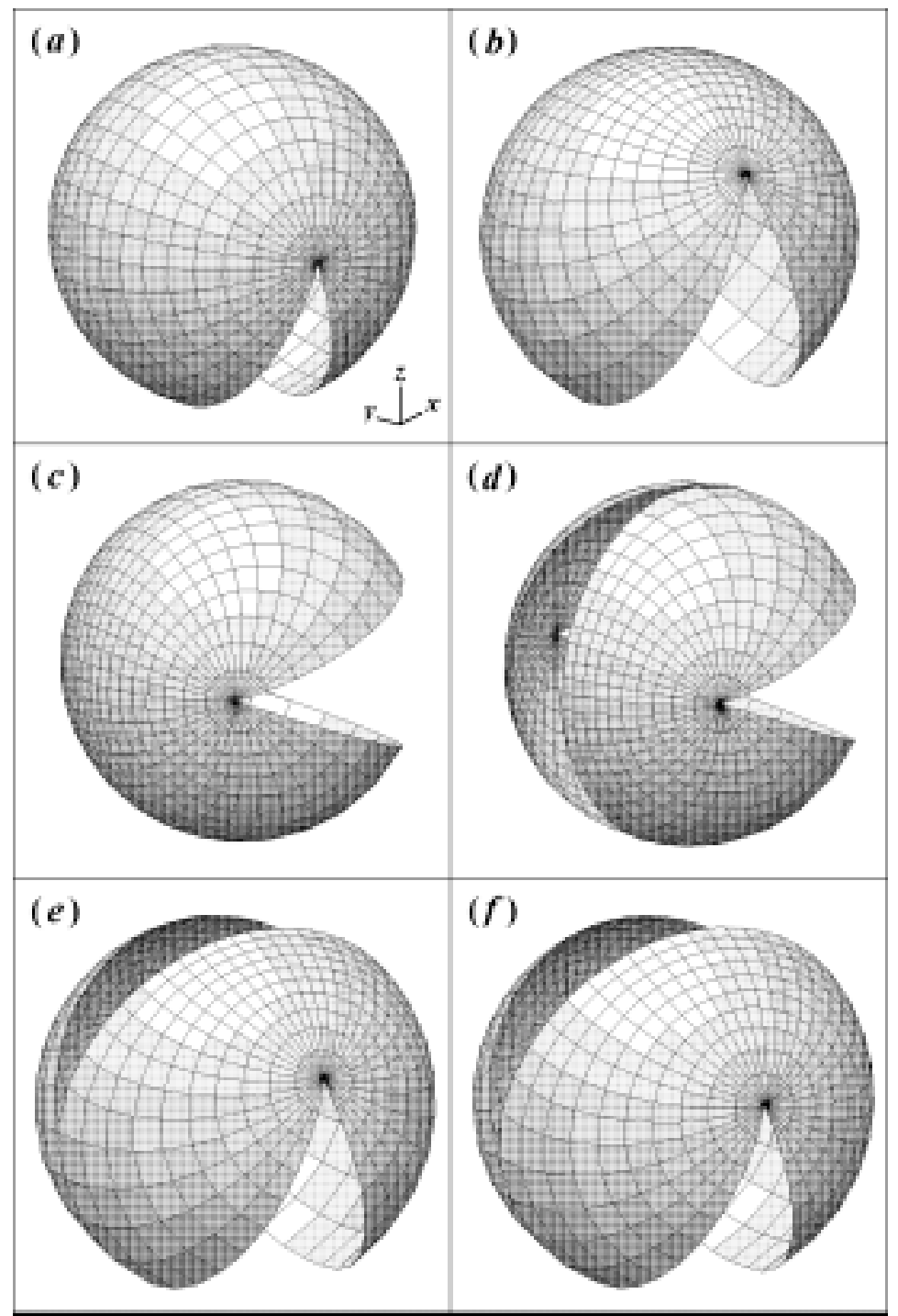

Figure 5: Representation on the Riemann sphere of the construction of the solution for two cosmic strings parallel to the $x$ and $y$-axes and moving apart in the positive and negative $z$ directions.

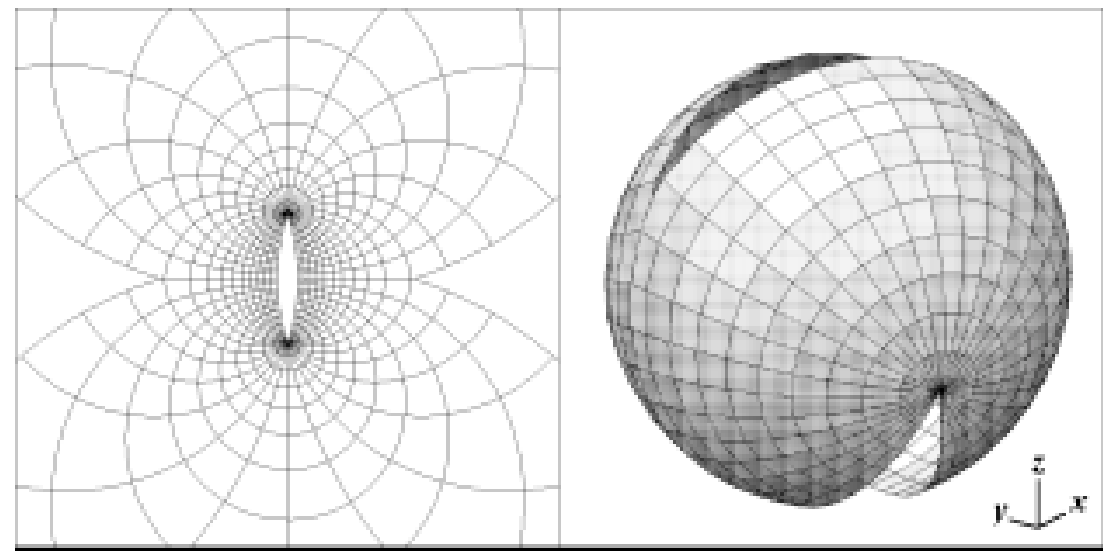

Figure 6: An alternative construction of the solution for two cosmic strings parallel to the $x$ and $y$-axes. The mapping $Z \rightarrow h_{2}(Z)$ given by Eqs. (22) and (25) in the complex Argand plane (left) corresponds to two cuts through the Riemann sphere (right) which are smaller than those in Fig. 5. 